This item was submitted to Loughborough's Research Repository by the author.

Items in Figshare are protected by copyright, with all rights reserved, unless otherwise indicated.

\title{
SQUID-arrays coupled to on-chip integrated thin-film superconducting input coils operating coherently
}

\section{PLEASE CITE THE PUBLISHED VERSION}

https://doi.org/10.1063/5.0032645

\section{PUBLISHER}

AIP Publishing

VERSION

AM (Accepted Manuscript)

\section{PUBLISHER STATEMENT}

This article may be downloaded for personal use only. Any other use requires prior permission of the author and AIP Publishing. This article appeared in Applied Physics Letters, 118 (4), 042601 (2021) and may be found at https://doi.org/10.1063/5.0032645.

\section{LICENCE}

\section{All Rights Reserved}

\section{REPOSITORY RECORD}

Chesca, Boris, Daniel John, and Robin Cantor. 2021. "Squid-arrays Coupled to On-chip Integrated Thin-film Superconducting Input Coils Operating Coherently". Loughborough University. https://hdl.handle.net/2134/13652942.v1. 


\title{
SQUID-arrays coupled to on-chip integrated thin-film superconducting input coils operating coherently
}

\author{
Boris Chesca ${ }^{a l}$, Daniel John ${ }^{a}$, and Robin Cantor ${ }^{b}$ \\ ${ }^{a}$ Department of Physics, Loughborough University, Loughborough LE11 3TU, United Kingdom \\ ${ }^{b}$ Star Cryoelectronics, Santa Fe, New Mexico 87508, USA
}

\begin{abstract}
Recently, Superconducting Quantum Interference Device (SQUID) arrays operating in a coherent voltage-modulation state at $77 \mathrm{~K}$ showed a flux-noise 10 times lower than single-SQUIDs at similar temperatures. To exploit SQUID-arrays flux-noise superiority in applications, however, it is essential to preserve the coherent state while the magnetic field to be measured, $B_{z}$, is highly inhomogeneous along the array as being generated by thin film integrated superconducting input coils or flux-transformers located in close proximity. Indeed, the flux coupled to each individual SQUID may vary significantly along the array, leading to a rapid degradation in the coherency. Here we present several solutions to avoid that based on a methodology we developed to assess the efficiency of signal coupling to SQUID-arrays while maintaining a highly coherent state. As a proof of concept, we applied it to highly-integrated $\mathrm{YBa}_{2} \mathrm{Cu}_{3} \mathrm{O}_{7} 800 / 770$ SQUID-arrays inductively coupled to on-chip integrated thin film superconducting input coils. Each SQUID in the array is directly coupled to two individual flux focusers leading to an increase in the effective area for which we derived an analytical formula. Consequently, we achieved SQUID-like voltage oscillation amplitudes above $10 \mathrm{mV}$ in the temperature range (75-83) $\mathrm{K}$ leading to a magnetic flux noise of $0.2 \mu \Phi_{0} / \mathrm{Hz}^{1 / 2}$, consistent with an ultra-enhanced coherent operation reached. For the
\end{abstract}

\footnotetext{
${ }^{1}$ Corresponding author; email: B.Chesca@lboro.ac.uk.
} 
strongest coupling scheme implemented experimentally a current white noise of $\mathrm{S}_{\mathrm{I}}{ }^{1 / 2}=32 \mathrm{pA} / \mathrm{Hz}^{1 / 2}$ was measured. This scheme can be used as the input coil of a flux-transformer resulting in a SQUID-array-based magnetometer with an estimated field sensitivity of $13 \mathrm{fT} / \mathrm{Hz}^{1 / 2}$.

The sensitivity of single-SQUID ${ }^{1-3}$ based magnetometers/gradiometers can be improved by building a series SQUID-array of $\mathrm{N}$ non-interacting SQUIDs operating in a coherent voltage modulation state. Indeed, it has been predicted ${ }^{4-6}$ that in this case the maximum SQUID-like voltage oscillation amplitude $\Delta V_{\max }$ linearly scales with $\mathrm{N}$, their dynamic range increases as $\mathrm{N}^{1 / 2}$, whereas white flux-noise $S_{\Phi}^{1 / 2}$ decreases as $1 / \mathrm{N}^{1 / 2}$. Consequently, the signal to noise ratio $\Delta V_{\max }$ $/ \mathrm{S}_{\Phi}{ }^{1 / 2}$ improves as $\mathrm{N}^{1 / 2}$. Much larger $\Delta V_{\max }$ means their matching for room temperature readout is greatly simplified. Coherent SQUID-arrays also have an improved bandwidth and impedance matching as their impedance is $\mathrm{N}$ times larger relative to a single SQUID. Coherence in SQUIDarrays made of $\mathrm{YBa}_{2} \mathrm{Cu}_{3} \mathrm{O}_{7}(\mathrm{YBCO})$ operating at $77 \mathrm{~K}$ has been first achieved in relatively small arrays $\mathrm{N}=30$ in the pioneering work $^{7}$ and later in $\mathrm{N}=10$ arrays connected to large area fluxfocusers $^{8}$. Earlier first attempts to operate large $\mathrm{N}$ (in the range 35-130) SQUID-arrays at $77 \mathrm{~K}$ did not show a coherent state throughout the entire array ${ }^{9,10}$. Recently coherent operation has been achieved $^{11}$ in highly integrated large arrays $(\mathrm{N}=484,770)$ at $77 \mathrm{~K}$ and above leading to an $1 / \mathrm{N}^{1 / 2}$ decrease for $S_{\Phi}{ }^{1 / 2}$ to below values of optimized single-SQUIDs with similar microns-size junction width operating at $4.2 \mathrm{~K}$. This result has two profound implications: first is the practicality of operation at $77 \mathrm{~K}$; second is that, due to its fundamental significance, SQUID-array's flux noise superiority can potentially translate into an improved field, current or energy sensitivities. This is because SQUID operation is based on the flux-quantization in a superconducting ring $^{1}$ and 
therefore SQUID's ultimate resolution as a sensor is fundamentally limited by the flux-noise $S_{\Phi}{ }^{1 / 2}$ that determines to which extent the flux-quantization phenomenon is deteriorated ${ }^{12}$. $\operatorname{In}^{11}$ the SQUID-arrays were inductively coupled to a 3-dimensional (3D) multiturn copper coil located 10 $\mathrm{mm}$ above which is not practical in applications due to a very weak coupling. Indeed, in most applications ${ }^{13} B_{z}$ is applied via on-chip integrated $2 D$ thin-film superconducting coils located in the near proximity, micrometres apart from the SQUIDs. Those signal coupling schemes/configurations ${ }^{13}$ were purposely developed for single-SQUIDs, which are small structures typically about $10 \mu \mathrm{m}$ in size, and variations of $B_{z}$ beyond this scale are irrelevant and have not been considered. However, $B_{z}$ changes significantly on a scale of a few millimetres, generating a large gradient along SQUID-arrays, which are 2D structures distributed over several millimetres. Consequently, the flux $\Phi_{i}$ coupled to each individual SQUID may vary significantly along the array, potentially leading to a rapid degradation in the coherency. Here we address two fundamental questions of what impact a large gradient of $B_{z}$ has on the degree of coherency in SQUID-arrays and how their response can be optimized. As a proof of concept of our approach/methodology two inductively signal coupling schemes implementing on-chip integrated superconducting thin-film input coils are considered theoretically and experimentally.

We consider a series array of $\mathrm{N}$ independent SQUIDs and implement the resistively-shuntedjunction model ${ }^{14}$ assuming that all SQUIDs in the array have identical Josephson junctions (of negligible width $W_{J J}$ and characterized by identical Josephson critical currents $I_{c}$ and normal resistances $R_{N}$ ) and inductances $L$, while the screening parameter $\beta_{L}=2 L I_{c} / \Phi_{0}$ is negligible $\left(\Phi_{0}\right.$ is the flux quantum). By generalizing the theory of single-SQUIDs ${ }^{14}$, the total voltage response $V$ of the SQUID-array to a magnetic field to be measured applied perpendicular to its planar structure, $B_{z}$, is a sum of all individual SQUID voltage oscillations: 


$$
V / \Delta V_{S Q U I D}=\sum_{j=1}^{N} \sqrt{\frac{I}{2 I_{c}}-\left|\cos \left(\pi \frac{\Phi_{j}}{\Phi_{0}}\right)\right|^{2}}
$$

Here $\Delta V_{S Q U I D}=I_{c} R_{N}$ is the amplitude of voltage oscillations of one SQUID, $I$, the dc bias current,

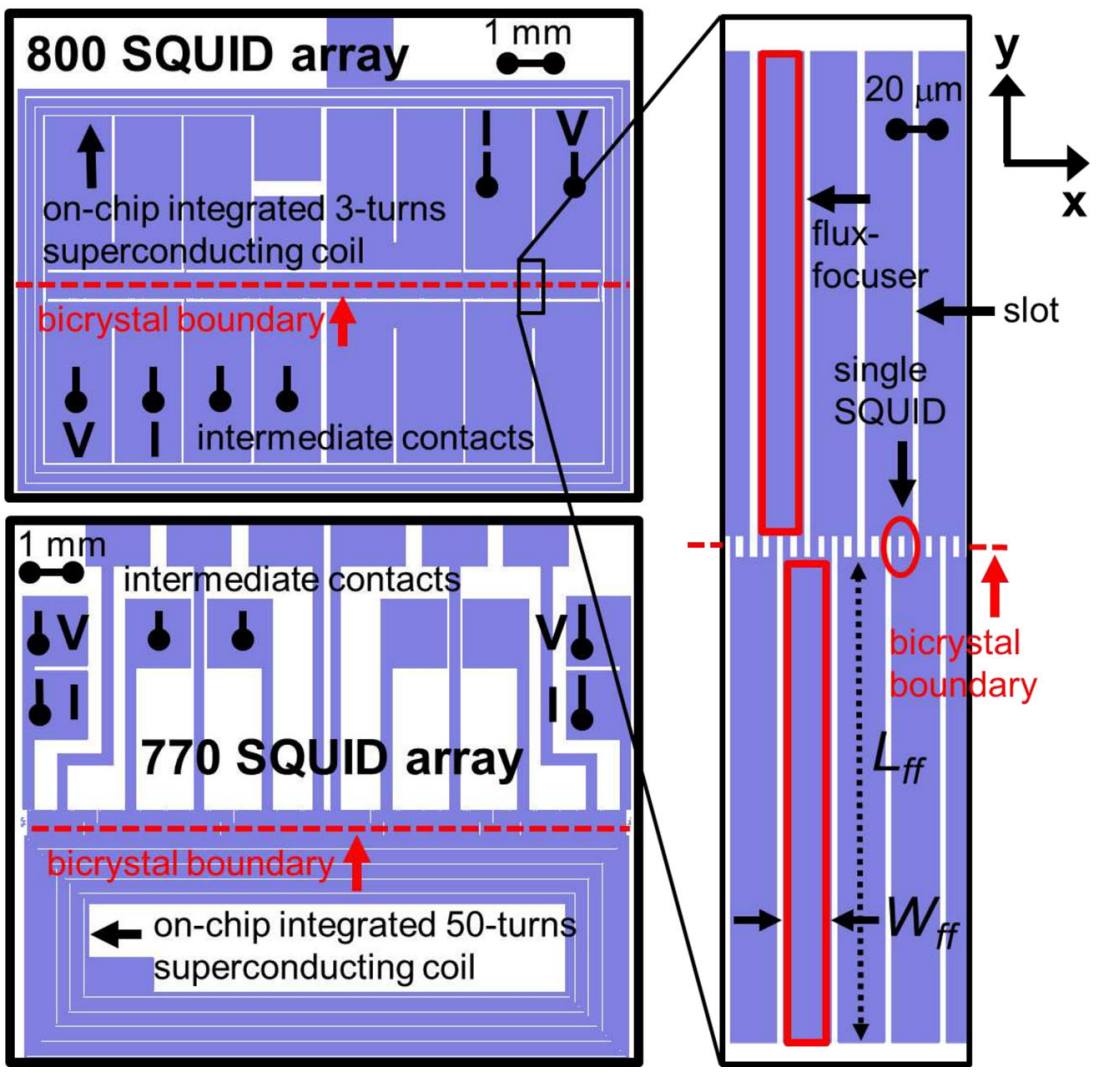

Fig.1. Layouts of the SQUID-arrays inductively coupled to on-chip integrated thin-film superconducting coils. Upper-left design: a 3-turns coil coupled to an 800 SQUID array placed inside the coil. Lower-left design: a 50-turns coil coupled via its outside edge to a 770 SQUIDarray located outside the coil; Right: enlarged view of a small central part of the 800 SQUID array showing 9 SQUIDs. Each SQUID has two Josephson junctions (seen as narrow bridges crossing the bicrystal boundary) and is directly connected to two large area narrow flux focusers (highlighted in red) having width $W_{f f}$ and length $L_{f f}$. 
and $\Phi_{j}$, the flux coupled to each individual SQUID, $j$, in the array. The effect on $V$ of a (5-10) $\%$ spread in the values of $I_{c}$ or $R_{N}$ has been theoretically considered elsewhere ${ }^{15-17}$. The SQUID-arrays distributed along an axis parallel to $x$ axis (see Fig.1) have been fabricated with a record level of integration of 100 SQUIDs/mm using standard bicrystal technology as described in $^{11}$. Each SQUID has two identical Josephson junctions connected in parallel in a rectangular loop configuration. The SQUIDs are connected in series in a serpentine path along the bicrystal grain boundary shown by the red dotted line. Two different designs (see Table 1) were fabricated and measured: an 800 SQUID-array and a 770-SQUID array. Each SQUID in the arrays is directly coupled to two long rectangular-shaped flux-focusers (ff) of length $L_{f f}$ and width $W_{f f}$ that

Table 1. SQUID-array fabrication and design parameters, electric transport measurements at $\mathrm{T}_{\max }$ (where the modulation amplitude reaches its maximum $\Delta V=\Delta V_{\max }$ ).

\begin{tabular}{|c|c|c|}
\hline SQUID-array & 800 SQUID-arrays & 770 SQUID-array \\
\hline $\begin{array}{l}\mathrm{SrTiO}_{3} \text { bicrystals } \\
\text { YBCO/Au films thickness } \\
\text { deposition method }\end{array}$ & $\begin{array}{l}30^{0} \text { symmetric }[001] \text { tilt } \\
200 \mathrm{~nm} \mathrm{YBCO} / 300 \mathrm{~nm} \mathrm{Au} \\
\text { reactive co-evaporation }\end{array}$ & $\begin{array}{l}24^{0} \text { symmetric }[001] \text { tilt } \\
100 \mathrm{~nm} \text { YBCO/200 nm Au } \\
\text { pulsed laser }\end{array}$ \\
\hline $\begin{array}{l}\text { Josephson junction's width, } W_{J J} \\
\left.\text { SQUID holes (length, } L_{h} \text {, width, } W_{h}\right) \\
\text { numerically calculated } L S Q U I D, W_{S Q U I D} \\
\text { calculated SQUID inductance, } L \\
\text { flux-focusers dimensions: }\left(L_{f f}, W_{f f}, S_{f f}\right) \\
\text { array length } \\
\text { flux focusing factor } A_{e f f, j} / A_{h, j}\end{array}$ & $\begin{array}{l}2 \mu \mathrm{m} \\
(8,3) \mu \mathrm{m} \\
(19.7,7) \mu \mathrm{m} \\
21 \mathrm{pH} \\
(180,17,3) \mu \mathrm{m} \\
8 \mathrm{~mm} \\
11.9\end{array}$ & $\begin{array}{l}3 \mu \mathrm{m} \\
(13,3) \mu \mathrm{m} \\
(34,9) \mu \mathrm{m} \\
30 \mathrm{pH} \\
(160,21,3) \mu \mathrm{m} \\
9 \mathrm{~mm} \\
16.1\end{array}$ \\
\hline $\begin{array}{l}\text { on-chip integrated input coil } \\
\text { linewidth } \\
\text { separation between turns } \\
\text { rectangle dimensions } \\
\text { calculated input coil inductance, } L_{i}\end{array}$ & $\begin{array}{l}3 \text {-turns rectangular spiral } \\
100 \mu \mathrm{m} \\
30 \mu \mathrm{m} \\
\text { minimum: } \\
\left(L_{\min }, W_{\min }\right)=(8.2,5.2) \mathrm{mm} \\
32 \mathrm{nH}\end{array}$ & $\begin{array}{l}50 \text {-turns rectangular spiral } \\
10 \mu \mathrm{m} \\
10 \mu \mathrm{m} \\
\text { maximum: } \\
\left(L_{\max }, W_{\max }\right)=(9.34,3.24) \mathrm{mm} \\
560 \mathrm{nH}\end{array}$ \\
\hline $\mathrm{T}_{\max }$ & $(81-83) \mathrm{K}$ & $(80-82) \mathrm{K}$ \\
\hline$\Delta V_{\max }$ & $10.1 \mathrm{mV}$ & $12.9 \mathrm{mV}$ \\
\hline$I_{c}, R_{N}, \beta$ (average across the arrays) & $44 \mu \mathrm{A}, 1.1 \Omega, 0.92$ & $90 \mu \mathrm{A}, 0.8 \Omega, 2.6$ \\
\hline$R_{\text {array }}$ & $0.44 / 0.22 / 0.11 \mathrm{k} \Omega$ & $0.31 \mathrm{k} \Omega$ \\
\hline $\begin{array}{l}\text { flux noise, } S_{\Phi}{ }^{1 / 2} \\
\text { current noise, } S_{I}{ }^{1 / 2}\end{array}$ & $\begin{array}{l}\text { Estimated }{ }^{*}: 0.26 \mu \Phi_{0} / \mathrm{Hz}^{1 / 2} \\
5.8 \mathrm{nA} / \mathrm{Hz}^{1 / 2}\end{array}$ & $\begin{array}{l}\text { Measured: } 0.2 \mu \Phi_{0} / \mathrm{Hz}^{1 / 2} \\
32 \mathrm{pA} / \mathrm{Hz}^{1 / 2}\end{array}$ \\
\hline
\end{tabular}

"assuming a similar voltage white noise as measured for the 770 SQUID-array. 
simultaneously ensure an increased field sensitivity and high levels of integration ${ }^{11}$. There is a narrow slot of width $s_{f f}$ between each pair of consecutive flux focusers strips. As for square-washer single-SQUIDs ${ }^{18-20}$, for each individual SQUID in the array there are significant flux-focusing effects expected leading to an increase in their effective pickup area $A_{\text {eff } j, j}$ relative to their respective rectangular hole area $A_{h, j}$ of length $L_{h}$ and width $W_{h}$. The difference between the two cases is that now the square-washers flux focusers ${ }^{18-20}$ are stretched out into long rectangles (strips) (see Fig. 1 and insets in Fig. 2). The main flux-focusing effect in SQUID-arrays can be understood within a model ${ }^{21,22}$ where two parallel thin film superconducting strips of width $W$ separated by a slot of width $s$ are placed in a perpendicular magnetic field $B_{z}$. In this geometry Meissner supercurrents concentrated near the edges of both strips (see red arrows in the left inset of Fig.2) will flow to screen the magnetic field $B_{z}$ from the superconducting strips. As a result of the field expelled from the strips, the field in the slot $B_{s l o t}$ is enhanced ${ }^{21,22}$ :

$$
\frac{B_{\text {slot }}}{B_{z}}=\frac{\pi k}{2 K\left(\sqrt{1-1 / k^{2}}\right)}
$$

Here $k=(2 W+s) / s$ and $K$ is the complete elliptic integral of the first kind. In our designs (see Fig.1) each SQUID in the array is surrounded by two pairs of parallel superconducting strips separated by a slot. Pair $(1,2)$ is located above the SQUID hole and pair $(3,4)$ below (see right inset in Fig. 2). Consequently, the field in the two slots located in the proximity of each SQUID hole is enhanced by a factor $B_{s l o t} / B_{z}$ given by Eq. (2) with $k=k_{f f}=\left(2 W_{f f}+s_{f f}\right) / s_{f f}$ calculated using flux-focuser dimensions. There is an additional flux focusing effect in SQUID-arrays that can be understood within a model ${ }^{18,23}$ where a thin film superconducting annular ring of outer and inner radii $a$ and $b$ is placed in a perpendicular magnetic field. In this geometry Meissner supercurrents concentrated near the hole and around the outside edge will flow to screen the magnetic field from the superconducting ring. As a result, the field inside the hole is enhanced ${ }^{18,19,23}$ by a factor 


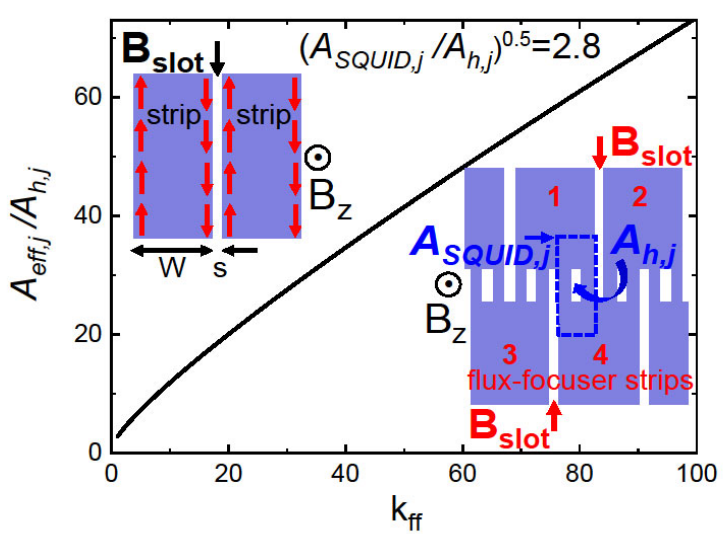

Fig.2. Increase in the normalized effective area $A_{e f f, j} / A_{h, i}\left(k_{f f}\right)$ given by Eq. (3). Left inset: the field is enhanced by $B_{\text {slot }} / B_{z}$ in a slot between two parallel superconducting strips in a perpendicular magnetic field $B_{z}$. Right inset: in SQUID-arrays the field is enhanced by $B_{s l o t} / B_{z}$ in the slot between each pair of flux-focusers strips (such as $(1,2)$ or $(3,4)$ ); a further field enhancement $B_{\text {eff }} / B_{\text {slot }}$ occurs inside the hole area of SQUID $j, A_{h, j}$.

approximately equal to $a / b$ (see Fig. 9 in $^{23}$ ). We performed numerical simulations ${ }^{24}$ based on the theory developed $\mathrm{in}^{23}$ and found that for rectangular shaped loops (SQUIDs) the field enhancement factor $B_{\text {eff }} / B_{\text {slot }}$ is equal to $\left(A_{S Q U I D, j} / A_{h, j}\right)^{0.5}$. Here $A_{s Q U I D, j}$ is the SQUID area rectangle (marked by dotted line in the right inset of Fig. 2) having a width $W_{S Q U I D}=2 \times W_{J J}+W_{h}$ and a length $L_{S Q U I D}$ determined from numerical simulations ${ }^{24}$ (see Table 1). This result is similar to the case of squarewasher SQUIDs ${ }^{18,19}$. Combining the two flux-focusing effects it follows that the effective pickup area $A_{e f f, j}$ of each SQUID, $j$, in the array increases as:

$$
\frac{A_{e f f, j}}{A_{h, j}}=\sqrt{\left(\frac{A_{S Q U I D, j}}{A_{h, j}}\right)} \frac{\pi k_{f f}}{2 K\left(\sqrt{1-1 / k_{f f}^{2}}\right)}
$$

$A_{e f f, j} / A_{h, j}\left(k_{f f}\right)$ given by Eq.(3) with $\left(A_{S Q U I D, j} / A_{h, j}\right)^{0.5}=2.8$ is plotted in Fig.2. Using data from Table 1 one finds $\left(k_{f f},\left(A_{S Q U I D, j} / A_{h, j}\right)^{0.5}\right)=(12.3,2.4)$ and $(15,2.8)$ leading to an increase for $A_{e f f, j} / A_{h, j}$ of 11.9 
and 16.1 for the 800 and 770 SQUID arrays, respectively. $\Phi_{j}$ can now be calculated by integrating $B_{z}(x, y, z)$ over $A_{e f f, j}$ area in the $(x, y)$ plane:

$$
\Phi_{j}=\iint_{A_{e f f, j}} B_{z}(x, y, z) d A
$$

A SQUID-array operates in a perfectly coherent voltage-modulation state when $\Phi_{j}$ given by Eq.(4) is constant along the array, $\left(\Phi_{j}=\Phi_{\text {const }}\right.$ for all SQUIDs $j$ ). In this case the SQUIDs-arrays are at their best against single-SQUIDs with their voltage oscillation amplitude $\Delta V$ being $\mathrm{N}$ times larger in Eq. (1): $V / \Delta V_{\text {SQUID }}=N \sqrt{I / 2 I_{c}-\left|\cos \left(\pi \Phi_{\text {const }} / \Phi_{0}\right)\right|^{2}}$.

We consider two inductive signal coupling schemes implementing on-chip integrated spiral rectangular superconducting thin-film input coils of inductance $L_{i}$ (see Fig. 1 and Table 1). First scheme is a 3-turns input coil coupled to an 800 SQUID-array placed inside. In this configuration the SQUIDs are located at different distances away from the coil with the first/last SQUIDs in the array being the closest at $50 \mu \mathrm{m}$. Second scheme is a 50-turns input coil strongly coupled via its outside edge to a 770 SQUID-array located outside the coil. Consequently, all SQUIDs in the array are located at the same distance from one edge of the coil with their centres at $180 \mu \mathrm{m}$ and their upper flux focusers edges at $10 \mu \mathrm{m}$. For the 800 SQUID-array $V$ was measured along the entire array or at intermediate contacts across smaller parts of the arrays in multiple sets of 200 SQUIDs. Accordingly, in the calculations, we varied $\mathrm{N}$ as well: $\mathrm{N}=200,400,800$.

To calculate the $z$ component of the magnetic field perpendicular to the SQUID-array plane $B_{z}$ generated by the current $I_{B}$ flowing through the input coils in the two coupling schemes considered we implement the Biot-Savart law for rectangular loops ${ }^{25}$. One can approximate a rectangular $M$-turns spiral coil as superposed multiple $M$ concentric rectangular loops with 
increasing length $L_{m}$ and width $W_{m} . B_{z}$ can then be derived from fields superposition generated by loop currents $I_{B}$ in each of the rectangular loops:

$$
B_{z}(x, y, z)=\frac{\mu I_{B}}{4 \pi} \sum_{m=1}^{M} \sum_{\alpha=1}^{4} \frac{1}{r_{\alpha}}\left[\frac{(-1)^{\alpha} y_{\alpha}}{r_{\alpha}+(-1)^{\alpha} x_{\alpha}}+\frac{x_{\alpha}}{r_{\alpha}+y_{\alpha}}\right]
$$

where $r_{1}=\sqrt{\left(x+L_{m} / 2\right)^{2}+\left(y+W_{m} / 2\right)^{2}+z^{2}}, r_{2}=\sqrt{\left(x-L_{m} / 2\right)^{2}+\left(y+W_{m} / 2\right)^{2}+z^{2}}$,

$$
r_{3}=\sqrt{\left(x-L_{m} / 2\right)^{2}+\left(y-W_{m} / 2\right)^{2}+z^{2}}, r_{4}=\sqrt{\left(x+L_{m} / 2\right)^{2}+\left(y-W_{m} / 2\right)^{2}+z^{2}},
$$

$-x_{1}=x_{4}=x+L_{m} / 2, x_{2}=-x_{3}=x-L_{m} / 2, y_{1}=y_{2}=y+W_{m} / 2$, and $y_{3}=y_{4}=y-$

$W_{m} / 2$.

Here $x, y$, and $z$ are the distances from the centre of the coils in the plane of the SQUID-array along both $x$ and $y$ axis, or in a direction perpendicular to the SQUID-array plane, respectively (see Figs.3a and $3 b$ ). Although in our experiments $z=0$ always, to understand how coherency changes with $z$, its value could be changed in the calculations. In Fig.3a $B_{z}(x, y, z)$ is plotted for the 3-turns coil for various $z$ as approximated by Eq.(5) with $\mathrm{M}=3$ rectangular loops with dimensions $\left(L_{m}, W_{m}\right)=(7.94+\mathrm{m} \times 0.26,4.94+\mathrm{m} \times 0.26) \mathrm{mm}$ and $\mathrm{m}=1,2,3$. For $\mathrm{z}=0 B_{z}(x, y=0, z=0)$ is highly inhomogeneous along the 800 SQUID-array increasing exponentially with $x$ from about 5 at $x=0$ (middle of array) to about 40 at $x=4$ (end of array; for better clarity the full range of this curve is not shown in Fig.3a). For $\mathrm{z}=0.5$ and $1 B_{z}(x, y=0, z=0.5)$ has a maximum, while for $z=2,3$, and 4 it decreases monotonically. In Fig. $3 \mathrm{~b} B_{z}(x=0, y, z=0)$ is plotted for the 50-turns coil as approximated by Eq.(5) with $\mathrm{M}=50$ rectangular loops with dimensions $\left(L_{m}, W_{m}\right)=(9.380-\mathrm{m} \times 0.04,3.280-\mathrm{m} \times 0.04)$ $\mathrm{mm}$ and $\mathrm{m}=1,2, . .50$. We found that $B_{z}(x, y=1.79, z=0)$ along the 770 SQUID-array axis is highly homogenous ( $1.79 \mathrm{~mm}$ is the $y$ coordinate of the centre of all SQUIDs). However, $B_{z}(x=0, y, z=0)$ is highly inhomogeneous along every single SQUID unit (consisting of the SQUID itself and the two flux-focusers) decreasing exponentially with $y$ (see Fig. 3b). By integrating $B_{z}(x, y, z)$ given by 
Eq. (5) using Eq.(4), $\Phi_{\mathrm{j}}$ can be calculated for each individual SQUID, $j$, in the array located at $\left(x_{j}, y_{j}\right)$. We have done this for both coupling schemes considered. Averaging the values of $\Phi_{\mathrm{j}}$ over the entire arrays $\left\langle\Phi_{j}\right\rangle_{\text {array }}$ we found that the flux couples more efficiently into the 770 SQUID-
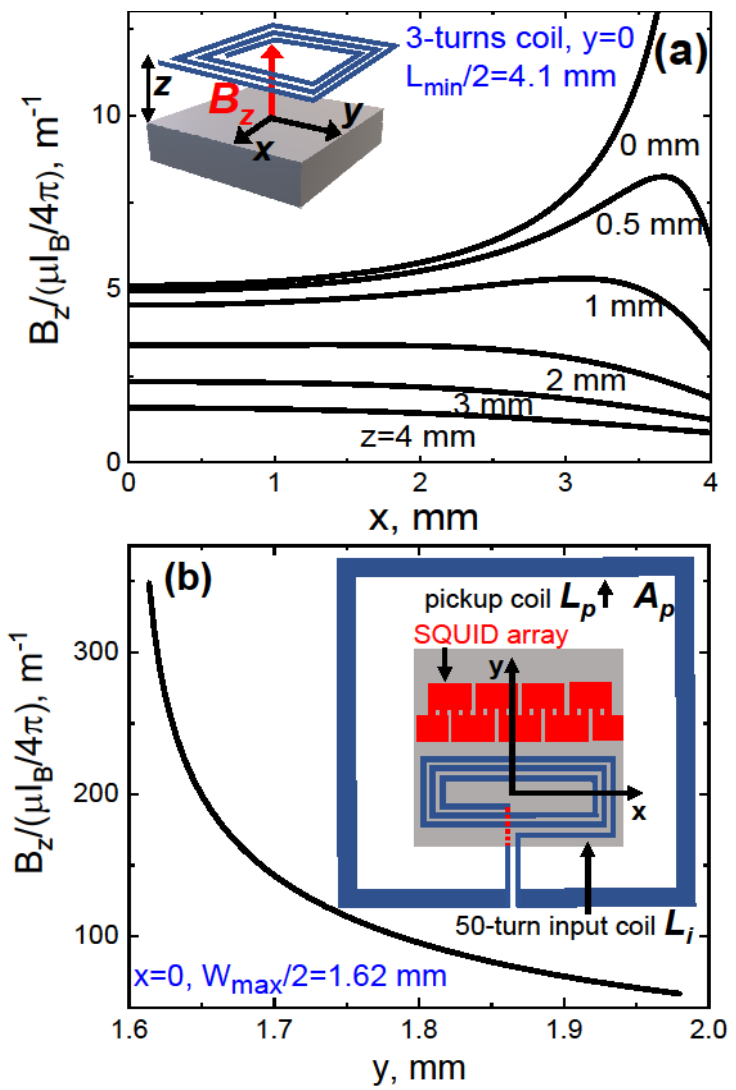

Fig.3. a) Normalized $B_{z}(x, y=0, z)$ along $x$-axis produced by a current $I_{B}$ through the 3-turns coil calculated with Eq. (5) for various $z$ (the distance between the coil and the SQUID-array plane) in the range $(0,4)$. b) Normalized $B_{z}(x=0, y, z=0)$ along $y$-axis produced by a current $I_{B}$ through the 50-turns input coil calculated with Eq.(5). Inset shows (not to scale) the schematics of a fluxtransformer consisting of a single turn, large area $A_{p}$ pickup coil of inductance $L_{p}$ and the 50-turn input coil of inductance $L_{i}$ inductively coupled to the SQUID array. 
array by a factor of $110=\left\langle\Phi_{j}\right\rangle_{770} /\left\langle\Phi_{j}\right\rangle_{800}$. $\Phi_{\mathrm{j}}$ values calculated above can finally be used in Eq.(1) to get the voltage response $V\left(I_{B}\right) / \Delta V_{S Q U I D}$ of the 800 SQUID-array (see Figs.4a, $4 \mathrm{~b}$ ). These calculations reveal several very interesting features as follow. The periodicity of $V / \Delta V_{S Q U I D}$ with $I_{B}$ monotonically increases with $z$ (due to $B_{z}$ decreasing with $z$ ), translating into a decrease in the device's signal sensitivity with $z$ (see Fig.4a). $\Delta V$ and its rate of decay with $I_{B}$ strongly depends on $z$ with an optimum response reached for $z_{o p t}=1.5 \mathrm{~mm}$ as in this case $B_{z}(x, y, z)$ has the highest degree of homogeneity along the array. At $z=0$ although the periodicity with $I_{B}$ is smallest (i.e., the sensitivity to $I_{B}$ is highest), the degree of coherence is worst, leading to a rapid decay of $\Delta V$ with $I_{B}$. Fig. $4 \mathrm{~b}$ shows the effect $\mathrm{N}$ has on the voltage response of the 800 SQUID-array coupled to the 3-turns coil when $\mathrm{z}=0 . \Delta V$ across the full array $(\mathrm{N}=800)$ decreases fast with increasing $I_{B}$ and, at its best, is only about 300 times larger than that of a single-SQUID. This is far less than 800 , the expected value for a perfectly coherent array. The voltage response across the middle 400 SQUIDs is very similar even though there are half as many SQUIDs. This is because the combined voltage response of the first 200 and the last 200 SQUIDs in the array is highly incoherent: those SQUIDs are distributed between $(-4,-2) \mathrm{mm}$ and $(2,4) \mathrm{mm}$ in a direction parallel to $x$ axis, where the inhomogeneity of $B_{z}$ is largest (see Fig. 3a). Consequently, $V\left(I_{B}\right) / \Delta V_{S Q U I D}$ has small oscillation amplitudes that are strongly modulated with $I_{B}$ while its periodicity with $I_{B}$ is relatively smaller. Consequently, their contribution does not interfere constructively with the response of the middle 400 SQUIDs. In fact, the voltage response of the middle 200 SQUIDs is almost as good as that of the full array for a similar reason. Decreasing $\mathrm{N}$ further below 200 leads to an approximately linear decrease in the oscillation amplitude with $\mathrm{N}$, as expected for coherent operation. Qualitatively similar results were obtained for non-coplanar coils $(z \neq 0)$. Since $B_{z}(x=0, y, z)$ decreases 

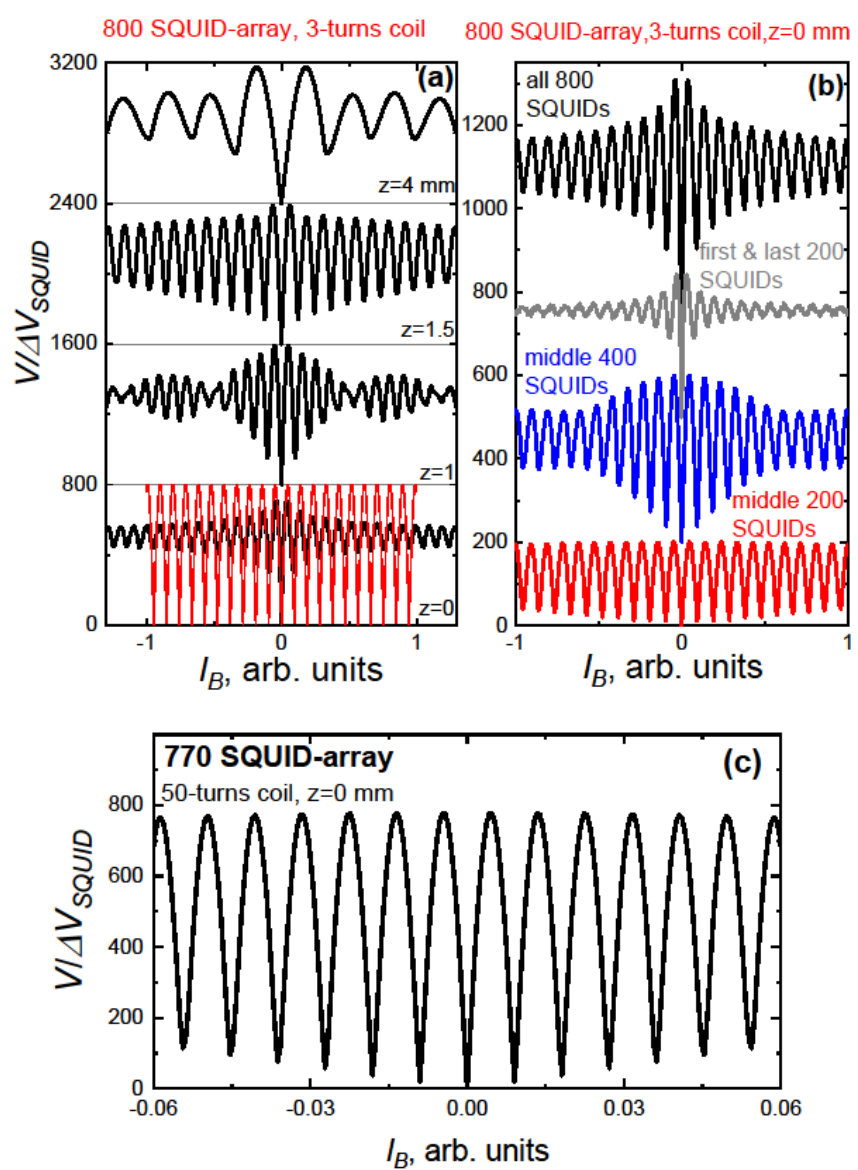

Fig.4. $V\left(I_{B}\right) / \Delta V_{S Q U I D}$ calculated with Eq.(1) (using Eq. (4) for $\Phi_{j}$ and Eq. (5) for $B_{z}$ ) and $\mathrm{I}=2 \mathrm{I}_{\mathrm{c}}$ for: a) the 800 SQUID-array coupled to the 3-turns coil and various $z$ (for clarity, the curves were shifted by multiples of 800); (b) the full array or various parts of it: middle 400 or middle 200 SQUIDs, as well as, the combined response of the first $200 \&$ last 200 SQUIDs in the array (for clarity, the curves were shifted vertically); (c) the 770 SQUID-array coupled to the 50-turns coil. The red curve in (a) is for a variable area $A_{e f f, j} 800$ SQUID-array to compensate for the highly inhomogeneous $\mathrm{B}_{\mathrm{z}}$ along the array. In this case a perfectly coherent operation is achieved with $\Delta V_{\max } / \Delta V_{S Q U I D}=800$. 
monotonically with $z$, a single-SQUID would be most sensitive if located at $z=0$ where $B_{z}$ is maximum. In contrast, a SQUID-array performs best if located not necessarily where $B_{z}$ is maximum but where a trade-off between a maximum for $B_{z}$ and a highest homogeneity for $B_{z}$ along the array is reached. To avoid any degradation in coherency in case $B_{z}$ is highly inhomogeneous

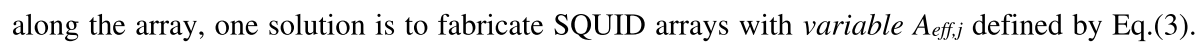
One should, however, avoid variations in either $A_{S Q U I D, j}$ or $A_{h, j}$ along the array as this leads to variations of $\beta_{L}$, which are known to supresses coherency ${ }^{11}$. Instead one should vary the flux focuser's parameter $k_{f f}=\left(2 W_{f f}+s_{f f}\right) / s_{f f}$ along the array in accordance to Eq. (3) so that $\Phi_{j}$, given by Eq.(4) (with $B_{z}(x, y, z)$ given by Eq.(5)), stays constant along the entire array. Thus, wider flux focusers or/and narrower slots are required in areas where $B_{z}$ is small.

One can avoid dealing with a highly inhomogeneous $B_{z}$ by implementing the other coupling scheme considered here. Indeed, one can place the SQUID-array outside a multi-turn rectangular spiral coil at $z=0$ in its proximity, with the array's axis parallel to one of the largest sides of the coil (see Fig.1). In this case, although $B_{z}$ decays exponentially along the $y$-axis (see Fig. 3b), it is highly homogeneous along the array. As before, for each SQUID $j$ located at $\left(x_{j}, y_{j}\right)$ of the 770 SQUID-array we first use $B_{z}(x, y, z)$ given by Eq.(5) into Eq.(4) to calculate $\Phi_{j}$ and then use Eq.(1) to get $V\left(I_{B}\right) / \Delta V_{S Q U I D}$. Thus, amplitudes of SQUID-like voltage oscillations as large as $97 \%$ of its perfectly coherent state value of 770 are produced (see Fig. 4c). Their magnitude decreases only slightly with increasing $I_{B}$ consistent with an ultra-enhanced coherent operation.

Families of dc current-voltage characteristics (IVC's) were measured for the 800 SQUID array at various temperatures in the range (70-89) $\mathrm{K}$ and multiple $I_{B}$. From such families of $I V C$ 's scanned over $I_{B}$, the dependence of the voltage modulation $V\left(I_{B}\right)$ for various bias currents $I$ could be constructed. Typical sets of measurements are shown in Figs.5a and $5 \mathrm{~b}$ with maximum 
amplitudes $\Delta V_{\max }$ of voltage oscillations around $10 \mathrm{mV}$. Remarkably, the amplitude of voltage oscillations of the middle 200 SQUIDs in the array $\left(\Delta V_{\max }=10.1 \mathrm{mV}\right.$ in Fig.5b) was not at all suppressed relative to the full array. In fact, the oscillation amplitude of $V\left(I_{B}\right)$ decreases only
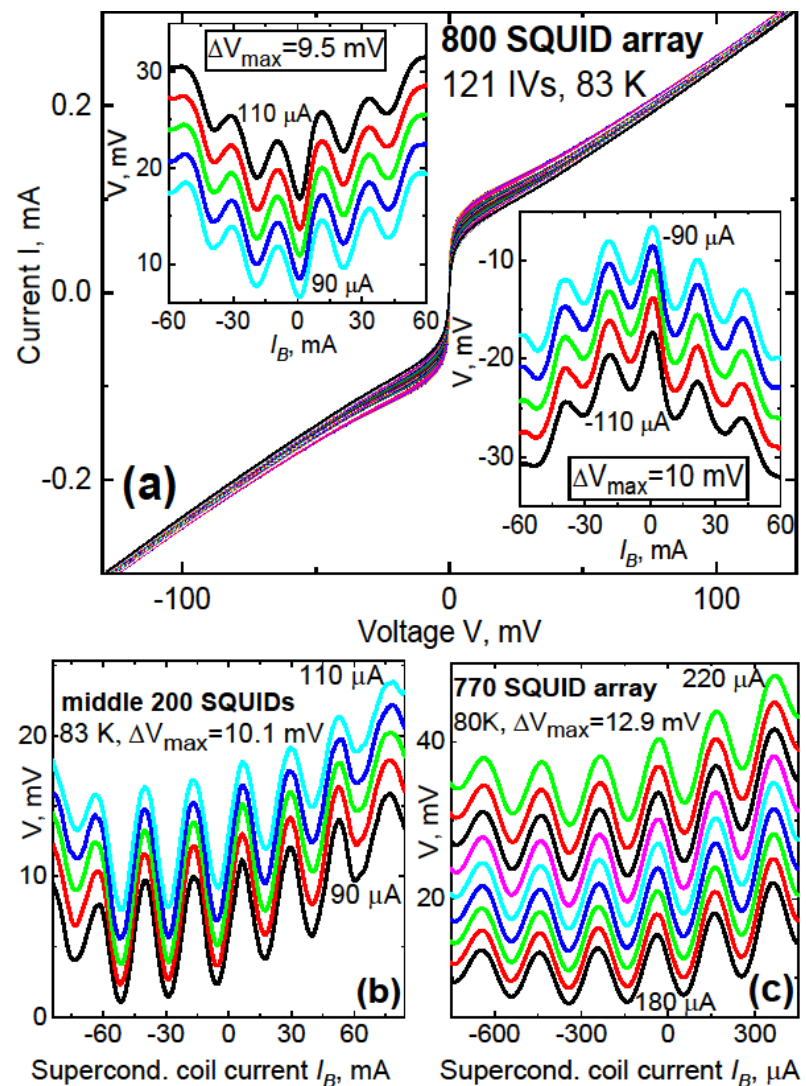

Fig.5. (a) 800 SQUID-array: a set of 121 consecutive IVC's at $83 \mathrm{~K}$ for various $B_{z}$ induced by $I_{B}$ (applied via a 3-turns superconducting coil) changed in steps of $1 \mathrm{~mA}$. Left/right insets: SQUIDlike oscillations of $V\left(I_{B}\right)$ for 5 different positive/negative bias currents $I$ in the range $( \pm 90, \pm 110)$ $\mu \mathrm{A}$. (b) 200 SQUID-array: $V\left(I_{B}\right)$ at $83 \mathrm{~K}$ for 5 different $I$ in the range $(90,110) \mu \mathrm{A}$. c) 770 SQUIDarray: $V\left(I_{B}\right)$ at $80 \mathrm{~K}$ with $I_{B}$ (applied via a 50-turns superconducting coil) changed in steps of $5 \mu \mathrm{A}$ for 9 different $I$ in the range $(180,220) \mu \mathrm{A}$. 
slightly with increasing $I_{B}$ suggesting a superior coherency reached relative to the full array. In this case the ratio $\Delta V_{\max } / \mathrm{N}=10.1 \mathrm{mV} / 200=50 \mu \mathrm{V} / \mathrm{SQUID}$ gets its largest value ever reported for SQUID-arrays operating above $77 \mathrm{~K}$. In contrast, the oscillation amplitude of $V\left(I_{B}\right)$ of the first 200 SQUIDs and the last 200 SQUIDs in the array were strongly suppressed to below $1 \mathrm{mV} . \Delta V_{\max }=9.7$ $\mathrm{mV}$ was measured for the middle 400 SQUIDs in the array at $83 \mathrm{~K}$, a value slightly larger relative to the full array. Qualitatively, this behaviour agrees well with our theoretical model (see Fig.4b).

Preliminary measurements of the 770 SQUID-array inductively coupled to a 3D copper coil located at $z=10 \mathrm{~mm}$ above showed ${ }^{11}$ SQUID-like oscillations with $\Delta V_{\max }=6.8 \mathrm{mV}$ at $83 \mathrm{~K}$ leading to a measured white voltage noise $S \mathrm{~V}^{1 / 2}=(8-9) n V / \mathrm{Hz}^{1 / 2}$. We have re-measured the 770 SQUID-array inductively coupled to the 50-turns superconducting coil (see Fig.1). The highest value for $\Delta V_{\max }$ of $12.9 \mathrm{mV}$ was obtained at a temperature of $80 \mathrm{~K}$ (see Fig.5c) leading to a magnetic flux noise $\mathrm{S}_{\Phi}{ }^{1 / 2}=\mathrm{Sv}^{1 / 2} /\left(\pi \Delta \mathrm{V}_{\max } / \Phi_{0}\right)=0.2 \mu \Phi_{0} / \mathrm{Hz}^{1 / 2}$. This increase in $\Delta V_{\max }$ relative to previous report ${ }^{11}$ is the consequence of an enhanced coherence state reached due to an improved degree of $B_{z}$ homogeneity along the array, in accordance with the theory (see Figs.4a and $4 c$ ).

Due to approximations made in our model there are quantitative differences between experiments and simulations: a drift/asymmetry of the measured $V\left(I_{B}\right)$ in Fig. 5 b and 5 c, as well as an enhanced amplitude modulation in the insets of Fig. 5a. In a more advanced model, one should consider non-zero values for both $W_{J J}$ and $\beta_{L}$, a superimposed remnant earth magnetic field in the cryostat, and an inhomogeneous distribution of Josephson critical currents along the array due to the non-uniformity of the bicrystal boundary. In this case there is no easy-to-use analytical solution, and the problem should be solved numerically.

The periodicity $\left(\Delta I_{B}\right)_{\Phi_{0}}$ of the measured $\mathrm{V}\left(I_{B}\right)$ corresponds to an additional flux quantum $\Phi_{0}$ coupled to each of the SQUDs in the array (see Figs.5a-5c). Consequently, the average mutual 
inductance $\left\langle M_{j}\right\rangle$ between the input coil and each individual SQUID $j$ can be calculated as $\left\langle M_{j}\right\rangle=\Phi_{0} /\left(\Delta I_{B}\right)_{\Phi_{0}}$. For the 3-turn input coil coupled to the 800 SQUID array, $\left(\Delta I_{B}\right)_{\Phi_{0}}=20 \mathrm{~mA}$ and $\left\langle M_{j}\right\rangle_{800}=0.1 \mathrm{pH}$ while for the 50-turns input coil coupled to the 770 SQUID-array $\left(\Delta I_{B}\right)_{\Phi_{0}}=160$ $\mu A$ and $\left\langle M_{j}\right\rangle_{770}=12.9 \mathrm{pH}$. The experimental value for the ratio $\left\langle M_{j}\right\rangle_{770} /\left\langle M_{j}\right\rangle_{800}=129$ agrees well with the theoretical value found earlier for the ratio of flux efficiency coupling $\left\langle\Phi_{j}\right\rangle_{770} /\left\langle\Phi_{j}\right\rangle_{800}=110$. This agreement further confirms the validity of our theoretical approach. A current noise sensitivity $\mathrm{SI}^{1 / 2}=\mathrm{S}_{\Phi}{ }^{1 / 2} /\left\langle M_{j}\right\rangle_{770}=32 \mathrm{pA} / \mathrm{Hz}^{1 / 2}$ is obtained for the 770 -SQUID array at $83 \mathrm{~K}$ which is about a factor 3 larger than for single-SQUIDs operated at $68 \mathrm{~K}^{26}$. To build a sensitive SQUID-array-based magnetometer, as for single-SQUIDs ${ }^{13,18-20}$, an inductively coupled flux transformer would be required to increase the effective area well beyond the flux focusing effects given by Eq.(3). This can be done by connecting the 50-turn input coil of inductance $L_{i}$ and area $A_{i}$ in a closed loop configuration to a single turn, large area $A_{p}$ square pickup loop of inductance $L_{p}$ fabricated on a separate substrate and placed on top of the SQUID-array substrate (see inset in Fig. 3b). We found that, similarly to single-SQUID magnetometers ${ }^{27}$, the total effective area of each SQUID $j$ operating as a magnetometer is:

$$
A_{m a g, j}=A_{e f f, j}+\left(A_{p}-A_{i}\right) \frac{\left\langle M_{j}\right\rangle_{770}}{L_{p}+L_{i}}
$$

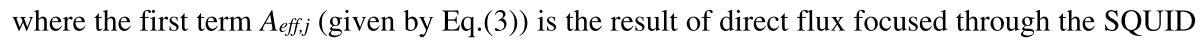
$j$ hole, while the second term represents the indirect flux due to the induced current in the input coil. To allow a direct comparison to single-SQUIDs from ${ }^{28}$ inductively coupled to fluxtransformers, we choose similar parameters for the pickup coil: $A_{p}=40 \times 40 \mathrm{~mm}^{2}=1.6 \times 10^{-3} \mathrm{~m}^{2}$ and a $2 \mathrm{~mm}$ linewidth. Using formulas from ${ }^{13}$ one gets: $L_{p}=80 \mathrm{nH}$ and $L_{i}=560 \mathrm{nH}$. Finally, using Eqs. (6) and (3) one finds $A_{m a g, j}=33 \times 10^{-3} \mathrm{~mm}^{2}$ which translates into an estimated field noise sensitivity 
for the 700 SQUID-array of $\mathrm{S}_{\mathrm{B}}{ }^{1 / 2}=\mathrm{S}_{\Phi}{ }^{1 / 2} / A_{\text {mag, } j}=13 \mathrm{fT} / \mathrm{Hz}^{1 / 2}$. This is more than a factor of two better relative to single-SQUIDs from ${ }^{28}$ having similar values for their SQUID inductance $(20-40 \mathrm{pH})$. Relative to the best reported values of $6 \mathrm{fT} / \mathrm{Hz}^{1 / 2}$ achieved in single SQUIDs with similar micronssize junction width inductively coupled to flux transformers and operating at $77 \mathrm{~K}^{29}$, our result is about a factor of two worse (note that in $^{29}$ the SQUID inductance is larger while $A_{p}$ is smaller). Since experimentally ${ }^{11} \mathrm{~S}_{\Phi}^{1 / 2}$ improves as $1 / \mathrm{N}^{1 / 2}$ we predict that, by increasing $\left\langle M_{j}\right\rangle$ to a few hundred $\mathrm{pH}, \mathrm{SQUID}$-arrays will outperform the best single-SQUID in terms of both $\mathrm{S}_{\mathrm{I}}{ }^{1 / 2}$ and $\mathrm{S}_{\mathrm{B}}{ }^{1 / 2}$. One possibility to increase $\left\langle M_{j}\right\rangle$ significantly is to place the input coil closer in a parallel plane located at a distance of (0.5-3) $\mu \mathrm{m}$ on top of the SQUID-array chip in a flip-chip configuration $^{8,13,20,26-29}$ (rather than in the same plane sideways: see inset of Fig. 3b).

In conclusion we have developed a methodology based on analytical solutions to assess and optimize the degree of voltage modulation coherency in SQUID-arrays inductively coupled to 2D on-chip integrated thin film superconducting input coils located in the near proximity that usually produce a highly inhomogeneous magnetic field along the array. Several solutions are presented: a) doubling the minimum size $L_{\min }$ of a coplanar $(\mathrm{z}=0)$ spiral coil relative to that of a SQUID-array placed inside in the area of maximum $B_{z}$ homogeneity; b) placing the SQUID-array outside a rectangular spiral coil in the near proximity (see Fig. $3 b$ ); c) placing a non-coplanar ( $z \neq 0)$ coil parallel to the SQUID-array plane at the optimal height zopt above (see Fig. 3a); d) coupling a coil located at $\mathrm{z}=0$ to SQUID-arrays with variable effective pickup area $A_{\text {eff } j}$ within the arrays. Consequently, SQUID-like voltage oscillation amplitudes above $10 \mathrm{mV}$ were measured in the temperature range (75-83) $\mathrm{K}$ leading to magnetic flux noises $\mathrm{S}_{\Phi}{ }^{1 / 2}=0.2 \mu \Phi_{0} / \mathrm{Hz}^{1 / 2}$. Our thin-film signal coupling schemes pave the way to fabricate ultra-sensitive SQUID-arrays-based magnetometers using inductively coupled flip-chip flux transformers. 
The research at Loughborough University (LU) was partially supported by the Higher Education Innovation Fund (HEIF) and Loughborough Enterprise Projects Group (EPG), LU code: S11519.

Data availability. The data that support the findings of this study are available from the corresponding author upon reasonable request.

\section{REFERENCES}

1. R. C. Jaklevic; J. Lambe; A. H. Silver, and J. E. Mercereau, Phys. Rev. Lett. 12 (7), 159 (1964).

2. D. Koelle, R. Kleiner, F. Ludwig, E. Dantsker, and J. Clarke, Rev. Mod. Phys. 71, 631 (1991).

3. The SQUID Handbook, Vol. I, Fundamentals and Technology of SQUIDs and SQUID Systems, Eds. J. Clarke and A. I. Braginsky, (Wiley-VCH, Weinheim, 2004).

4. R.P. Welty and J.M. Martinis, IEEE Trans. Magn. 3, 2924 (1991).

5. R.P.Welty and J.M. Martinis, IEEE Trans. Appl. Supercond., 3, 2605 (1993).

6. K.G. Stawiasz and M.B. Ketchen, IEEE Trans. Appl. Supercond. 3, 1808 (1993).

7. K. Li, S.P. Hubbell, R. Cantor, M. Teepe, IEEE Trans. Appl. Supercond., 9, 4420 (1999).

8. S. H. Wu, M.H. Hsu, K.L.Chen, J.C. Chen, J.T. Jeng, T.S. Lai, H. R. Horng, H. C. Yang, Supercond. Sci. Technol. 19, S246 (2006).

9. S. G. Lee, Y. Huh, G.S. Park, I.S. Kim, Y. K. Park, J.C. Park, IEEE Trans. Appl. Supercond. 7, 3347 (1997).

10. C. H. Wu, M. J. Chen, J. C. Chen, K. L. Chen, H. C. Yang, M. S. Hsu, T. S. Lai, Y.S. Tsai, H. E. Horng, J. H. Chen, J. T Jeng, Rev. Sci. Instrum.77, 033901 (2006).

11. B. Chesca, D. John, C. Mellor, Appl. Phys.Lett. 107162602 (2015);

12. B. Chesca, IEEE Trans. Appl. Supercond. 9, 2955 (1999).

13. R. Cantor and D. Koelle, chapter 5: Practical DC SQUIDS: Configuration and Performance, in The SQUID Handbook, Vol. 1, Eds. J. Clarke and A. I. Braginsky, (WileyVCH, Weinheim, 2004).

14. B. Chesca, D. Koelle, and R. Kleiner, Chapter 2: SQUID theory in The SQUID Handbook, Vol. 1, Eds. J. Clarke and A. I. Braginsky (Wiley-VCH, Weinheim, 2004).

15. J. T. Jeng, H. C. Hung, C. R. Lin, C. H. Wu, K. L. Chen, J.-C. Chen, H. C. Yang, S. H. Liao, and H. E. Horng, IEEE Trans. Appl. Supercond., 15, 793, (2005).

16. S. M. Wu, Shane A. Cybart, S. M. Anton, and R. C. Dynes, IEEE Trans. Appl. Supercond., 23, 1600104, (2013)

17. D. Crété , Y. Lemaître, B. Marcilhac, E. Recoba-Pawlowski, J. Trastoy and C. Ulysse, J. Phys. Conf. Series 1559, 012012 (2020). 
18. M. B. Ketchen, W. J. Gallagher, A. W. Kleinsasser, S. Murphy, J. R. Clem, SQUID'85, Superconducting Quantum Interference Devices and Their Applications, Ed. H. D. Hahlbohm and H.Lübbig (Berlin: De Gruyter) 865 (1981).

19. J. M. Jaycox and M. B. Ketchen, IEEE Trans. Magn. 17, 400 (1981).

20. A. H. Miklich, J. J. Kingston, F. C. Wellstood, J. Clarke, M. S. Colclough, K. Char, and G. Zaharchuk, Appl. Phys. Letts. 59, 988 (1991).

21. A. A. B. Brojeny, Y. Mawatari, M. Benkraouda, and J. R. Clem, Supercond. Sci. Technol. 15, 1454 (2002).

22. A. A. B. Brojeny and J. R. Clem, Supercond. Sci. Technol. 17, 1275 (2004).

23. A. A. B. Brojeny and J. R. Clem, Phys. Rev. B 68, 174514 (2003).

24. B. Chesca, unpublished;

25. M. Misakian, J. Res. Natl. Inst. Stand. Technol. 105, 557 (2000).

26. Blomgren, T. Eriksson, and D. Winkler, IEEE Trans. Appl. Supercond., 11, 892 (2001).

27. B. Oh, R.H. Koch, W.J. Gallagher, R.P. Robertazzi, and W. Eidelloth, Appl. Phys. Letts. 59, 123 (1991).

28. D. Koelle, A. H. Miklich, E. Dantsker, F. Ludwig, D. T. Nemeth, J. Clarke, W. Ruby, and K. Char, Appl. Phys. Letts. 63, 3630 (1993).

29. M. I. Faley, U. Poppe, K. Urban, D. N. Paulson, T. Starr and R.L. Fagaly, IEEE Trans. Appl. Supercond. 11, 1383 (2001). 


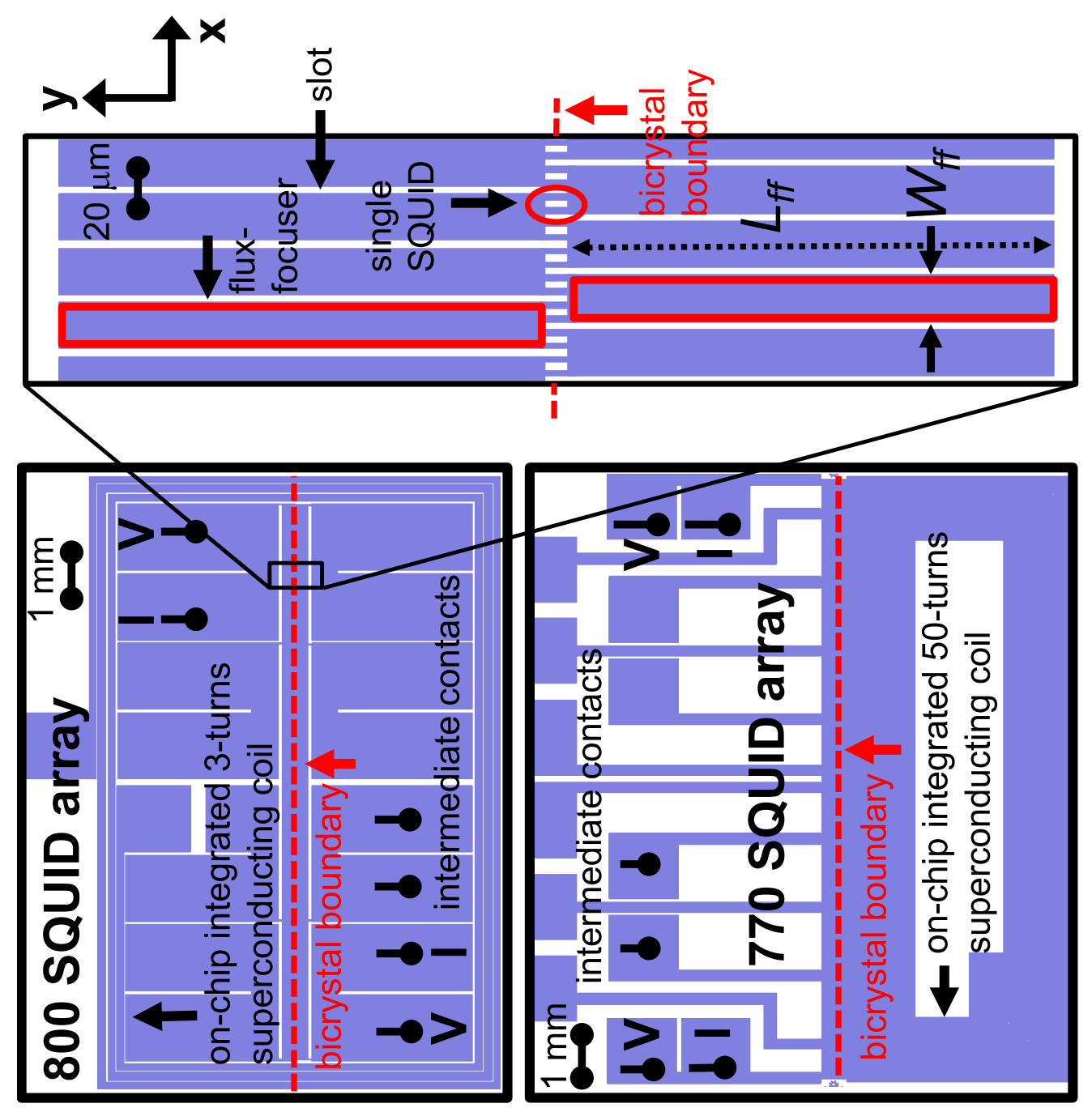

吕畺 


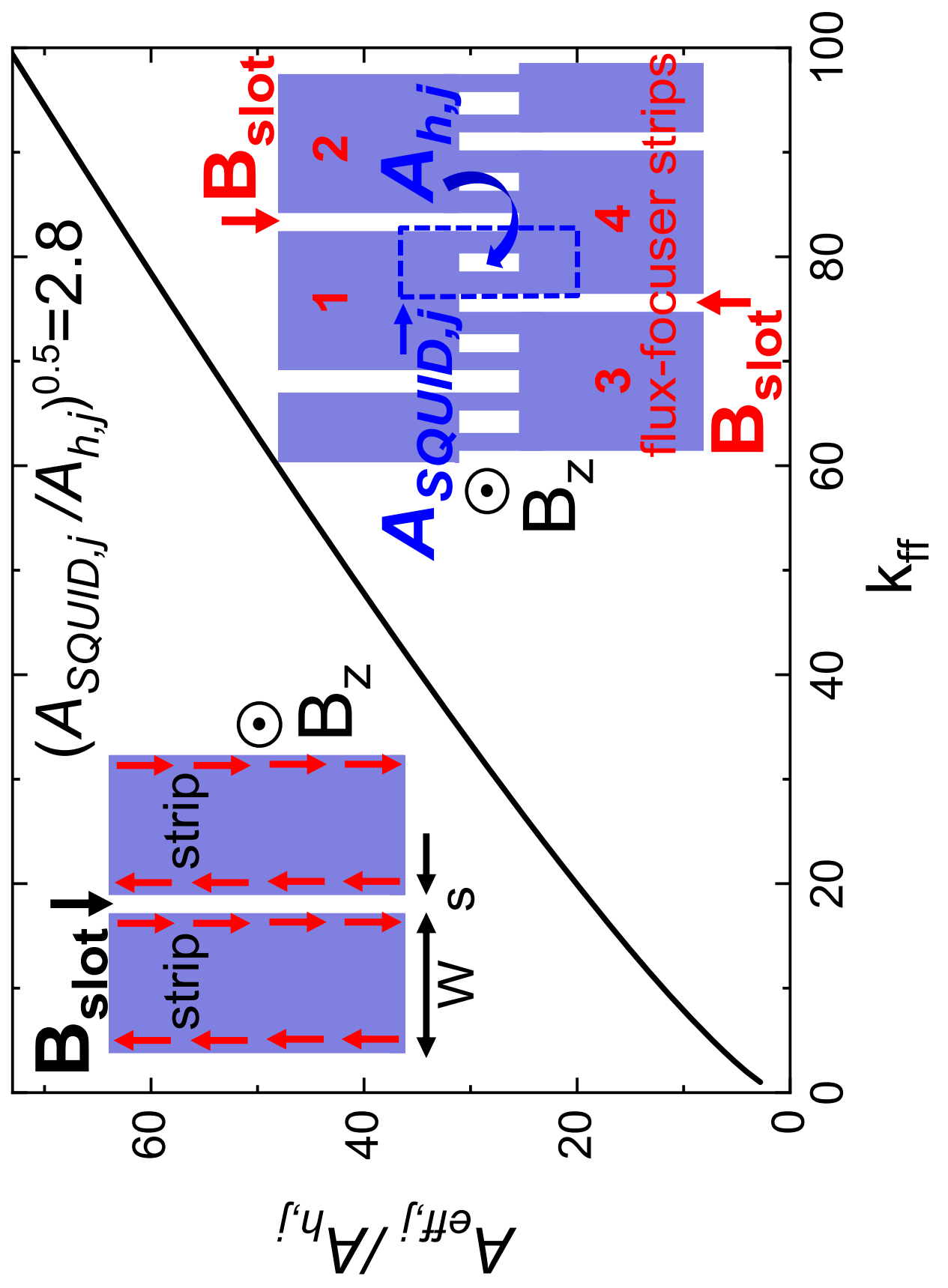

兄耪高 

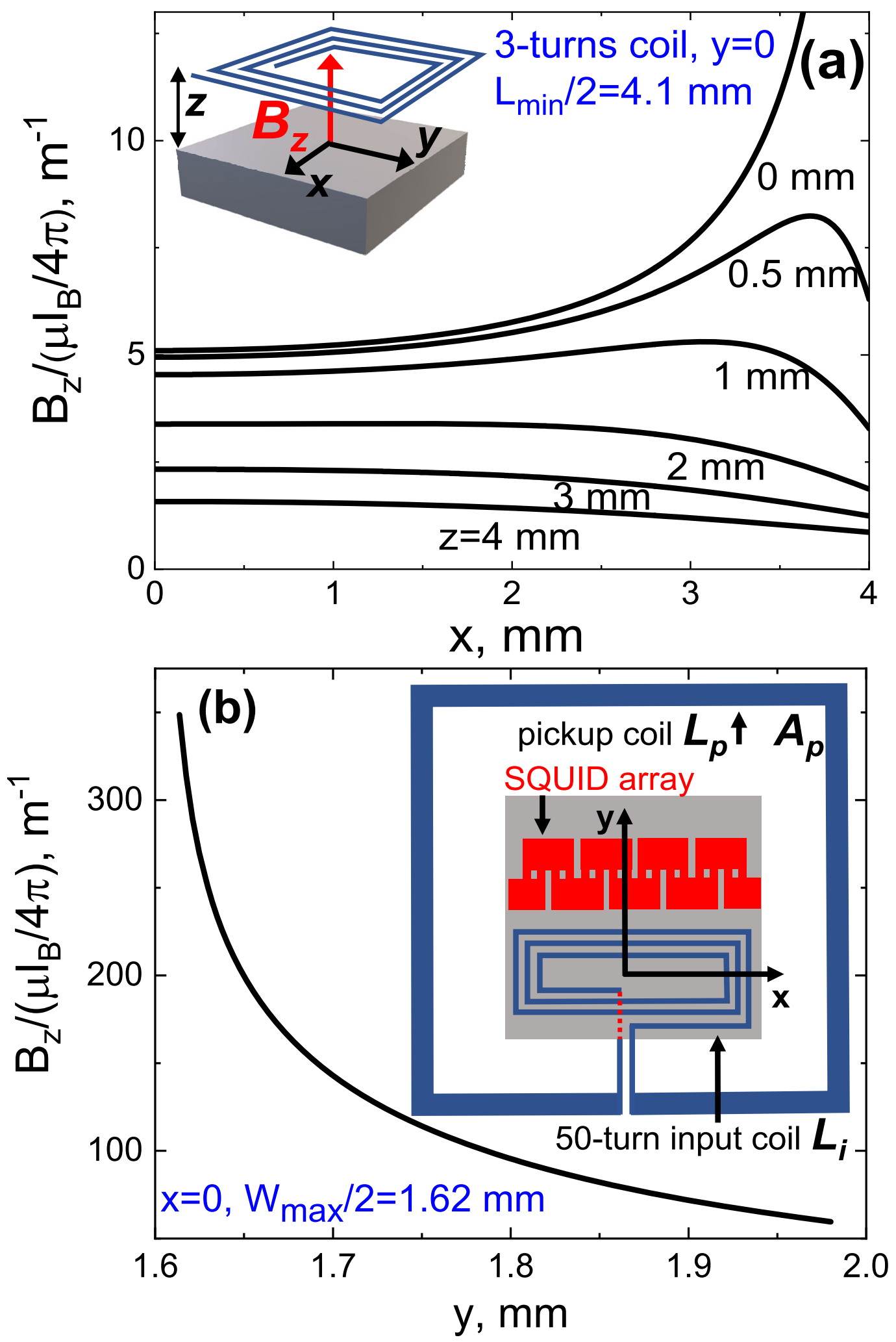
800 SQUID-array, 3-turns coil 800 SQUID-array,3-turns coil,z=0 mm
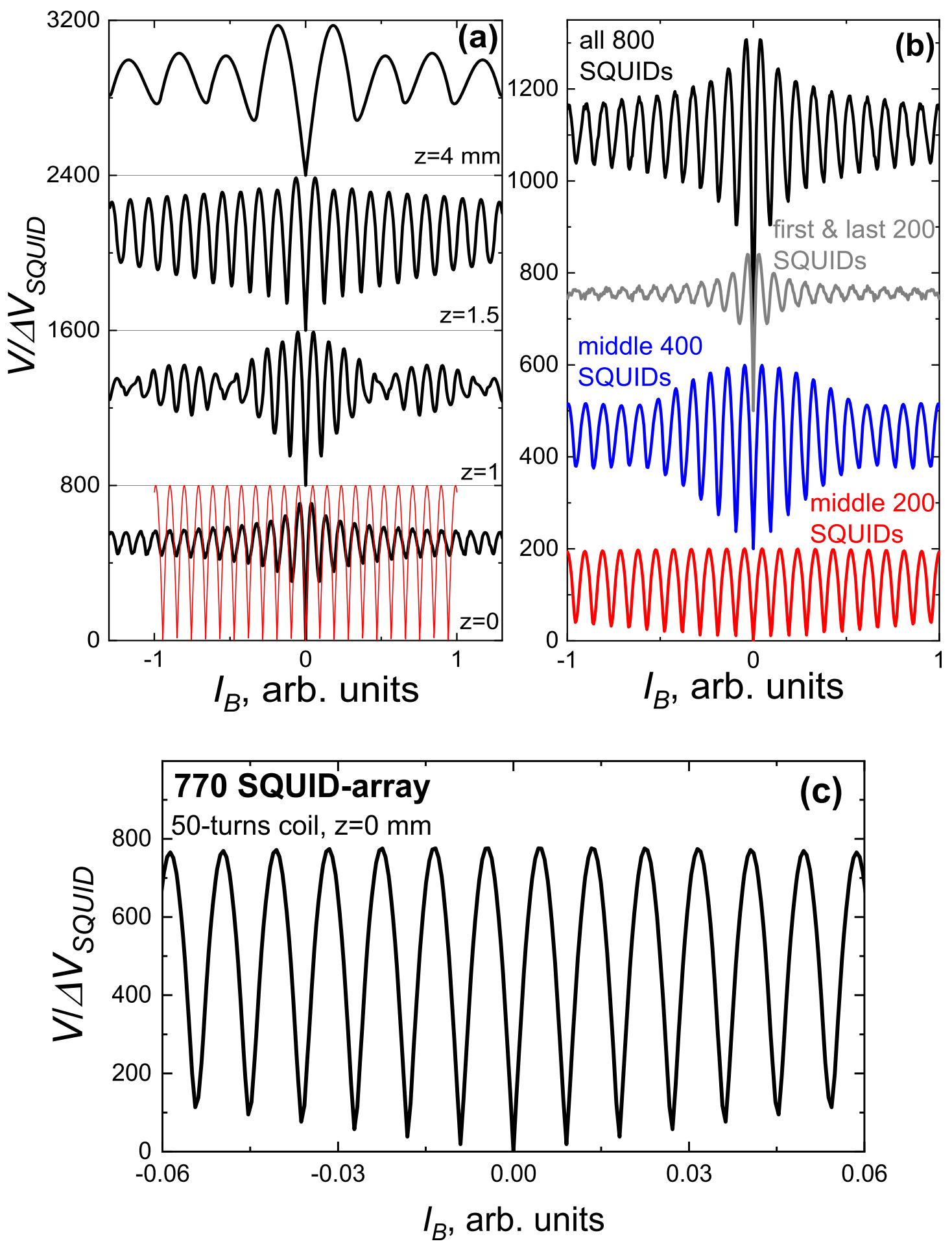

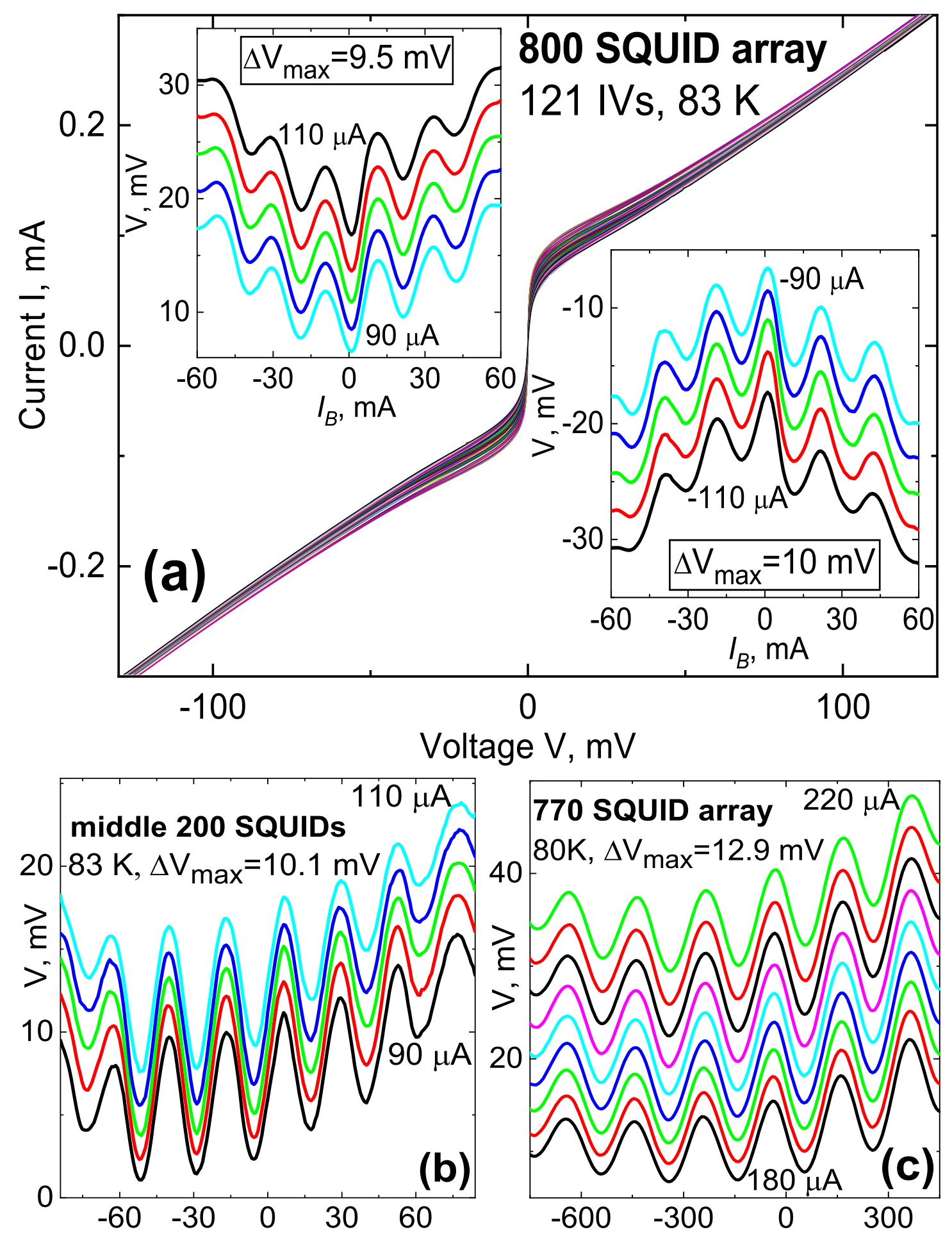

Supercond. coil current $I_{B}, \mathrm{~mA}$ Supercond. coil current $I_{B}, \mu \mathrm{A}$ 